\title{
Relación entre la precipitación registrada en estados reproductivos de la soja y la severidad de Septoria glycines y Cercospora kikuchii
}

\author{
Marcelo Carmona ${ }^{1}$, Ricardo Moschini ${ }^{2}$, Graciela Cazenave ${ }^{2} \&$ Francisco Sautua $^{1}$ \\ ${ }^{1}$ Cátedra de Fitopatología, Facultad de Agronomía, Universidad de Buenos Aires, C1417DSE, Buenos Aires, Argentina; \\ ${ }^{2}$ Instituto Nacional de Tecnología Agropecuaria - INTA, Instituto de Clima y Agua, Las Cabaña y Los Reseros, CC 25 \\ (1712), Castelar, Argentina
}

Autor para correspondencia: Marcelo Carmona, e-mail: carmonam@agro.uba.ar

\section{RESUMEN}

Durante los años 2003 a 2008 se evaluó la severidad de las enfermedades de fin de ciclo (SevEFC, mancha marrón y tizón morado) en el estado fenológico R7 en localidades de las provincias de Santa Fe y Córdoba. Los valores de SevEFC $(\mathrm{N}=15)$ se categorizaron binariamente en función de un valor umbral en: epidemia severa (Sev EFC $>36 \%$ ) y epidemia moderada a ligera (<=36 \%). La variación de la SevEFC en R7 fue analizada en relación a variables ligadas a la precipitación, procesadas entre R3 y R5. Las variables se expresaron como frecuencia y precipitación acumulada en mm, que superan los umbrales: 1, 5, 7 y $10 \mathrm{~mm}$. Las variables DPr7 (días con precipitación $>7 \mathrm{~mm}$ ), PrAc7 (acumulado de precipitaciones diarias $>7 \mathrm{~mm}$ ) y la interacción entre ambas (It7) resultaron ser las más fuertemente correlacionadas con los niveles epidémicos (coeficiente de Kendall Tau-b $\left(\mathrm{r}_{\mathrm{K}}=0,74,0,60\right.$ y 0,71 respectivamente). El modelo de regresión logística que incluyó a PrAc7 estimó correctamente la probabilidad de ocurrencia en 12 de las 15 observaciones de SevEFC. Los modelos logísticos que integraron las variables DPr7 o It7, obtuvieron precisiones de predicción de 93,3\%. Estos resultados podrían ser útiles para la predicción y control de las EFC.

Palabras clave: Glycine max, enfermedades de fin de ciclo, modelos logísticos.

\begin{abstract}
Relationship between precipitations registered in reproductive stages of soybean and severity of Septoria glycines and Cercospora kikuchii

From 2003 to 2008, late season disease severity (LSDsev) values (Brown spot and Cercospora leaf blight) were recorded at the R7 growth stage at several sites in Santa Fe and Córdoba Provinces. The annual LSDsev records $(\mathrm{N}=15)$ were grouped into two epidemic categories based on a threshold value (median of observed disease data): severe (LSDsev $>36 \%$ ) and moderate to light (LSDsev $<=36$ $\%)$. Variations in epidemic levels were studied in relation to precipitation-based variables, processed in a time window limited by R3 and R5 growth stages. The variables were expressed as frequency (days) and total accumulation (mm) of daily precipitations greater than the thresholds 1, 5, 7 and $10 \mathrm{~mm}$. The variables PrF7 (number of days with precipitations $>7 \mathrm{~mm}$ ) and AcPr7 (total accumulation of daily precipitations $>7 \mathrm{~mm}$ ) and the interaction (product) between them (It7) were the most strongly correlated, according to Kendall tau-b coefficients $\left(r_{K}=0.74,0.60\right.$ and 0.71 respectively). Logistic regression model including AcPr7 correctly estimated the probability of occurrence of epidemic categories in 12 cases (out of 15). Logistic models integrating PrF7 or the interaction effect (It7) presented prediction accuracies of $93.3 \%$. These results could be useful for prediction and chemical control of LSD.
\end{abstract}

Keywords: Glycine max, late season diseases, logistic models.

\section{INTRODUCCIÓN}

Durante la campaña 2008/09, la Argentina alcanzó una siembra record de 17 millones de hectáreas de soja, constituyéndose en la mayor superficie sembrada en la historia del país con una única especie (SAGPyA, 2008). Casi la totalidad de la siembra correspondió a cultivares modificados genéticamente y la mayor parte se cultiva bajo siembra directa (SD). Este proceso de crecimiento estuvo acompañado por el avance de la frontera agrícola, el monocultivo y la siembra de genotipos cada vez más adaptados, siendo además, fuertemente orientado por la rentabilidad económica que ofrece. Entre las limitantes más importantes que presenta el cultivo en todas las regiones, deben mencionarse las enfermedades, especialmente las foliares cuya prevalencia e intensidad se incrementan considerablemente cada año. La combinación indeseable del monocultivo y SD, genera excelentes condiciones para la multiplicación y supervivencia de los patógenos causantes de las llamadas enfermedades de fin de ciclo (EFC), que son actualmente las más graves para el cultivo por los daños que puede causar. Varios estudios (Carmona et al., 2004; Carmona, 2006) estimaron daños promedio en soja en Argentina por efecto de las EFC que 
oscilaron entre $8 \%$ y $10 \%$, con un máximo individual de $30 \%$.

Las EFC de mayor prevalencia en Argentina son: tizón de la hoja y mancha púrpura de las semillas (Cercospora kikuchii (Tak. Matsumoto \& Tomoy.) M.W. Gardner), antracnosis (Glomerella glycines, Colletotrichum truncatum), mancha marrón (Septoria glycines), tizón de la vaina y tallo (Phomopsis sojae), mildiu (Peronospora manshurica), mancha ojo de rana (Cercospora sojina Hara), mancha anillada (Corynespora cassiicola), mancha foliar por Alternaria (Alternaria spp.), pústula bacteriana (Xanthomonas campestris pv glycines) y tizón bacteriano (Pseudomonas siringae pv glycinea). La mayoría de los patógenos causantes de las EFC son necrotróficos (extraen nutrientes de tejidos muertos). De manera similar a lo que ocurre con la mayoría de los necrotróficos de otros cultivos, los agentes causantes de las EFC sobreviven en semillas y rastrojo (Hartman et al., 1999).

El estudio epidemiológico de las EFC muestran que si bien los síntomas que causan se tornan más conspicuos en etapas reproductivas intermedias y avanzadas del cultivo, la mayoría de los patógenos que las provocan están presentes en etapas previas (Klingelfuss \& Yorinori, 2001), en algunos casos provocando infecciones latentes sin posibilidad de observación directa a campo (asintomáticas) con prolongados periodos de incubación o formando estructuras reproductivas que contribuyen a aumentar la presión de inóculo en las etapas de mayor susceptibilidad de la planta. Como consecuencia de lo anterior hay un aumento de la severidad hacia el fin de ciclo que incluso se puede confundir con la senescencia natural, pudiendo muchas veces pasar desapercibido, lo que dificulta la toma de decisión (Carmona, 2006). Estas características son de gran importancia debido a que la espera de la visualización de los síntomas y fructificaciones (como puede ser costumbre para otras enfermedades en cultivos como trigo, maíz o cebada) puede llevar a un atraso en la toma de decisión de control químico, en relación al período crítico de generación de rendimiento del cultivo de soja, no logrando el impacto deseado (Carmona et al., 2009).

En este contexto, probablemente las precipitaciones serían las que más ayudarían a definir la respuesta a la aplicación de fungicida. Por eso, fungicidas aplicados para el control de las EFC en períodos de sequía no incrementan significativamente el rendimiento, ya que estas enfermedades no se desarrollan en esas condiciones epidemiológicas (Backman et al., 1984). La mayoría de los patógenos causantes de las EFC, a excepción de Cercospora, presentan fructificaciones hidrofílicas (picnidios, acérvulos) que necesitan de agua para el proceso de diseminación (liberación, dispersión y deposición del inóculo) (Maude, 1996; Carmona et al., 2009). Asimismo, el mayor número y frecuencia de horas de mojado también explicarían el incremento de la intensidad de todas las EFC (Lim, 1980; Schuh, 1993). En relación a la temperatura, las condiciones térmicas que se registran durante el crecimiento del cultivo de soja en la región pampeana $\left(15-30^{\circ} \mathrm{C}\right)$, aseguran condiciones predisponentes para la infección de la mayoría de los hongos causantes de las EFC (Lim, 1980; Schuh \& Adamowicz, 1993; Schuh, 1993; Hartman et al., 1999), siendo probablemente las precipitaciones y el mojado foliar, las condiciones más importantes para su intensidad. Este trabajo tuvo como objetivo determinar la relación cuantitativa entre variables hídricas (simples e interacciones) procesadas entre las fechas de R3 y R5 (Fehr et al., 1971) y la intensidad de las EFC en R7 expresada en severidad foliar.

\section{MATERIALES Y MÉTODOS}

\section{Monitoreo y cuantificación de las enfermedades}

Durante los años 2003 a 2008 se realizaron observaciones de severidad de las EFC, principalmente mancha marrón (S. glycines) y tizón morado (C. kikuchii), en el estado fenológico R7 de variedades susceptibles de soja sembradas en varias localidades del sur de Santa $\mathrm{Fe}$ (S.Fe) y Sudeste de Córdoba (Cba) (Tabla 1). La distancia promedio entre establecimientos fue de $23 \mathrm{~km}$. Los lotes que, además de las mencionadas manchas foliares, presentaban otras enfermedades tales como bacteriosis, no fueron incluidos para su evaluación. Para cuantificar la severidad de las enfermedades, se estimó visualmente el porcentaje de área foliar clorótica, necrosada o con síntomas, avaliando de cada lote, 30 plantas tomadas al azar a lo largo de un transecto. Para proceder a la cuantificación, las plantas fueron divididas en tres tercios (superior, medio e inferior). La severidad de cada enfermedad fue estimada visualmente considerando el área foliar con necrosis y clorosis respecto al área foliar total de cada tercio. Los valores de cada tercio fueron promediados para obtener la severidad promedio de cada enfermedad. Para obtener el valor final de severidad de las EFC se promediaron las observaciones de ambos patógenos. En los casos donde C. kikuchii tuvo valores nulos, el valor de severidad de las EFC resultó ser el de $S$. glycines. Se ha utilizado esta metodología ya que si bien C. kikuchii no estuvo presente en todos los ensayos, es uno de los patógenos predominantes en Pradera Pampeana, por lo que la inclusión de su efecto en el análisis es de suma importancia.

La metodología de determinar severidad estimando visualmente elárea foliar afectada es aplicada frecuentemente por diversos autores (Niero et al., 2007; Fernandez et al., 2002; Diaz et al. 2005; da Costa 2005). Con el fin de determinar y confirmar los patógenos involucrados en la sintomatología, se incubó el material vegetal con síntomas, previamente desinfectado con hipoclorito al $1 \%$ durante 1 minuto, en cámara húmeda a temperatura entre 24 y $27^{\circ} \mathrm{C}$, con alternancia de 12 horas de luz cercana al ultravioleta y 12 horas de oscuridad. En cada uno de los ensayos se midieron las precipitaciones con pluviómetros ubicados en cada uno de los establecimientos, registrando su valor diario. 
TABLA 1 - Valores observados de severidad de las EFC (SevEFC) en R7, discriminando según los correspondientes a los patógenos $S$. glycines (Sept) y C. kikuchii (Cerc) y su promedio (EFC). También por campaña, sitio y variedad de soja se presentan las fechas (en día juliano: Djul) de los estados fenológicos R3 y R5

\begin{tabular}{|c|c|c|c|c|c|c|c|}
\hline \multirow[t]{2}{*}{ Campaña } & \multirow[t]{2}{*}{ Sitio } & \multirow[t]{2}{*}{ Variedad } & \multirow{2}{*}{$\begin{array}{c}\text { R3 } \\
\text { Djul }\end{array}$} & \multirow{2}{*}{$\begin{array}{c}\text { R5 } \\
\text { DJul }\end{array}$} & \multicolumn{3}{|c|}{ Severidad en R7 } \\
\hline & & & & & Sept & $\begin{array}{c}\text { Cerc } \\
\%\end{array}$ & EFC* \\
\hline $2003 / 04$ & Armstrong (S.Fe) & AW4303 & 26 & 48 & 22 & 0 & 22 \\
\hline $2003 / 04$ & Armstrong (S.Fe) & DM4800 & 21 & 52 & 37 & 30 & 33 \\
\hline $2003 / 04$ & Arteaga (S.Fe) & AW3901 & 36 & 60 & 17 & 0 & 17 \\
\hline $2003 / 04$ & Montes Oca (S.Fe) & DM4800 & 24 & 53 & 36 & 22 & 29 \\
\hline $2003 / 04$ & Godeken (S.Fe) & DM4400 & 22 & 47 & 34 & 0 & 34 \\
\hline $2004 / 05$ & Armstrong (S.Fe) & DM3700 & 23 & 40 & 41 & 0 & 41 \\
\hline $2004 / 05$ & Armstrong (S.Fe) & DM4800 & 41 & 61 & 35 & 38 & 37 \\
\hline $2004 / 05$ & Armstrong (S.Fe) & MSYNG & 29 & 48 & 46 & 0 & 46 \\
\hline $2004 / 05$ & Arteaga (S.Fe) & DM4800 & 33 & 62 & 37 & 0 & 37 \\
\hline $2005 / 06$ & Armstrong (S.Fe) & DM4800 & 24 & 44 & 47 & 37 & 42 \\
\hline $2005 / 06$ & Armstrong (S.Fe) & DM4800 & 44 & 62 & 37 & 35 & 36 \\
\hline $2005 / 06$ & Monje (S. Fe) & A 5901 & 47 & 66 & 40 & 37 & 38 \\
\hline $2005 / 06$ & Cruz Alta (Cba) & DM3700 & 24 & 43 & 38 & 34 & 36 \\
\hline $2005 / 06$ & M. Juárez (Cba) & N4725 & 48 & 65 & 41 & 34 & 37,5 \\
\hline $2007 / 08$ & Las Parejas (S.Fe) & N4725 & 29 & 49 & 26 & 0 & 26 \\
\hline
\end{tabular}

* Solo en las observaciones con valores nulos de C. kikuchii se supuso que la superficie foliar ocupada por S. glycines representó por sí sola la severidad de la EFC

\section{Desarrollo de los modelos}

Variable dependiente: probabilidad de ocurrencia de niveles categorizados de severidad de enfermedades de fin de ciclo (SevEFC) asociadas a $S$. glycines (Sept) y $C$. kikuchii (Cerc).

Categorización: los valores de severidad de las EFC (SevEFC, Tabla 1) se categorizaron binariamente en función de un valor umbral (mediana de los valores observados) en: epidemia severa (S: SevEFC $>36 \%$ y epidemia moderada a ligera (M: SevEFC $<=36 \%$ ). Esta categorización aplicada basada en la mediana, tuvo objetivos prácticos y didácticos.

Variables meteorológicas regresoras: la variación en los niveles de severidad de la EFC en R7 fue analizada en relación a las siguientes variables hídricas (simples e interacciones), procesadas entre las fechas de R3 y R5: variables simples: DPr1: número de días con registro de precipitación> $1 \mathrm{~mm}$; PrAc1: precipitación acumulada $(\mathrm{mm})$, del total de los registros de lluvia diaria $>1 \mathrm{~mm}$; DPr5 y PrAc5: idem para registros diarios de lluvia $>5 \mathrm{~mm}$; DPr7 y PrAc7: idem para registros diarios de lluvia $>7 \mathrm{~mm}$. DPr10 y PrAc10: idem para registros diarios de lluvia $>10$ $\mathrm{mm}$. Interacciones: producto de 2 variables simples: It1 $=\mathrm{DPr} 1 * \operatorname{PrAc} 1 ; \quad$ It5 $=\mathrm{DPr} 5 * \operatorname{PrAc} 5 ; \quad$ It $7=\mathrm{DPr} 7 * \operatorname{PrAc} 7 ;$ It10 $=\mathrm{DPr} 10 * \operatorname{PrAc} 10$

Análisis estadístico

Cálculo de coeficientes de correlación $\left(r_{k}\right)$ de Kendall Tau-b (Proc Freq del SAS) entre los niveles binarios epidémicos y las variables meteorológicas ligadas a las precipitaciones descritas (simples e interacciones).
Las variables ambientales con más alta correlación con los niveles de infección fueron utilizadas para desarrollar los modelos de regresión logística.

Regresión logística (De Wolf et al., 2002; Moschini et al., 2006): el procedimiento Logistic del SAS ajusta modelos de regresión para datos de respuesta binaria por medio del método de máxima verosimilitud. Una función logit (logaritmo natural de (PrS / 1- PrS)) establece la unión entre el componente estocástico y las variables regresoras meteorológicas, siendo PrS la probabilidad de una epidemia severa (S), es decir, de superar al valor umbral de severidad de las EFC (mediana $=36 \%$ ). El modelo logístico, con $\mathrm{X}_{1}$ como predictor y $\beta_{0}$ y $\beta_{1}$ los parámetros, puede escribirse como: $\ln (\operatorname{Pr} S / 1-\operatorname{PrS})=\beta_{0}+\beta_{1} X_{1}$. Resolviendo la expresión: $1 /\left(1+\operatorname{Exp}^{-(\ln (\operatorname{PrS} / 1-\operatorname{PrS})}\right)$, se obtiene el valor de $\operatorname{PrS}$. La probabilidad de una epidemia moderada a ligera (M) se calcula como 1 - PrS. Si para un año y sitio específico (analizados en Tabla 1) el valor PrS predicho por la ecuación es $>0,5$, entonces el nivel epidémico se clasifica como $\mathrm{S}$ (Severo), estimándose que la severidad de las EFC es > al valor umbral (36\%, mediana). En la evaluación final de los mejores modelos logísticos se toma en cuenta el valor crítico $\mathbf{P}$ (valor de probabilidad para clasificar a un caso como $\mathrm{S}$ que logra la mayor precisión de predicción), que no necesariamente es el valor 0,5 . Derivado del análisis de regresión logística se calcularon para cada modelo ajustado la sensibilidad ( $\%$ de observaciones epidémicas $>$ al valor umbral mediana de severidad correctamente predichas como S), especificidad ( $\%$ de observaciones epidémicas $<=$ al umbral correctamente clasificadas como $\mathrm{M}$ ), falsos $\mathrm{S}$ y 
falsos M. Sensibilidad y especificidad de los modelos fueron usados para calcular la precisión de predicción (Hughes \& Madden, 2003).

Se analizó el efecto discriminante de las variables que integran los modelos seleccionados, comparando los valores que alcanzan las variables en el primer cuartil (25\% de los años), mediana (50\%) y tercer cuartil (75\%) para el grupo de años categorizados como $\mathrm{S}(\mathrm{N}=7)$ versus los observados por el grupo de años categorizados como $\mathrm{M}$ $(\mathrm{N}=8)$.

\section{RESULTADOS}

Los registros individuales por sitio y variedad de la severidad observada de $S$. glycines (Sept) y de C. kikuchii (Cerc), en el estado fenológico R7 se muestran en la Tabla 1. La mancha marrón estuvo presente en el $100 \%$ de los lotes involucrados con un rango de severidad de $17-46 \%$, mientras que el tizón morado sólo en el $53 \%$ de los mismos con valores de severidad de $0-38 \%$, (Tabla 1). Todas las variedades involucradas en el relevamiento resultaron susceptibles al ataque de las EFC. Los resultados de las incubaciones realizadas en laboratorio, indicaron que las fructificaciones y estructuras de los patógenos observados coincidieron con la sintomatología registrada en campo.

En la Tabla 2 se presentan los coeficientes de correlación (r) de Kendall Tau-b entre los niveles epidémicos y las variables meteorológicas simples e interactivas analizadas. Las variables DPr1, PrAc1 y su interacción, que incluyen el impacto de lloviznas y lluvias ligeras (precipitaciones diarias a partir del umbral de $1 \mathrm{~mm}$ ), alcanzaron los menores valores de correlación de Kendall. Ambas variables calculadas con precipitaciones mayores a 7 mm (DPr7 y PrAc7) y su interacción (It7) alcanzaron los máximos coeficientes de Kendall.

Con estos 3 factores (DPr7, PrAc7 y It7) se ajustaron las ecuaciones de los modelos logísticos (Tabla 3) para estimar la probabilidad de ocurrencia de niveles epidémicos categorizados como severos (S). Para cada modelo se detallan los valores de sensibilidad y especificidad, con los cuales se calculó la precisión de predicción (\%), o sea el porcentaje de casos correctamente clasificados.

El modelo I que incluyen a PrAc7 clasificó correctamente 12 de las 15 observaciones de severidad de las EFC (1 falso S y 2 falsos M). Los modelos II y III, que incluyen a la variable DPr7 y al componente interactivo It7 (DPr7*PrAc7) respectivamente, permitieron predecir correctamente 14 casos de los 15 observados (93,3\%

TABLA 2 - Coeficientes de correlación (r) de Kendall Tau-b de variables meteorológicas en relación a los niveles epidémicos de la FET (severo y moderado-ligero)

\begin{tabular}{lllll}
\hline \hline Variables & \multicolumn{4}{c}{ Coeficiente de Correlación (r) de Kendall } \\
\hline Simples & DPr $1=0,54$ & DPr5 $=0,62$ & DPr7 $=0,74$ & DPr10=0,60 \\
& $\operatorname{PrAc} 1=0,58$ & $\operatorname{PrAc5}=0,60$ & $\operatorname{PrAc7}=0,60$ & $\operatorname{PrAc} 10=0,58$ \\
Interactivas & It $1=0,60$ & It $5=0,60$ & It $7=0,71$ & It $10=0,54$ \\
\hline
\end{tabular}

DPr1: número de días con registro de precipitación $>1 \mathrm{~mm}$. PrAc1: precipitación acumulada $(\mathrm{mm})$ del total de los registros de 1 luvia diaria $>1 \mathrm{~mm}$. DPr5 y PrAc5: idem para registros diarios de lluvia $>5 \mathrm{~mm}$. DPr7 y PrAc7: idem para registros diarios de lluvia $>7 \mathrm{~mm}$. DPr10 y PrAc10: idem para registros diarios de lluvia $>10 \mathrm{~mm}$. It1=DPr1*PrAc1; It5=DPr5*PrAc5; It7=DPr7*PrAc7; It10=DPr10*PrAc10.

TABLA 3 - Modelos logísticos basados en variables meteorológicas para estimar la probabilidad (PrS) de ocurrencia de un nivel de severidad de EFC severo (S: SevEFC $>36 \%$ ). P: valor crítico de probabilidad para clasificar a un caso epidémico como S. Sensibilidad (Sens): \% de observaciones epidémicas $>36 \%$ correctamente predichas como S. Especificidad (Esp): $\%$ de observaciones epidémicas $<=$ $36 \%$ correctamente predichos como moderado-ligero $(\mathrm{M})$. La precisión de predicción representa el \% de casos epidémicos analizados $(\mathrm{N}=15)$ correctamente clasificados como $\mathrm{S}$ y $\mathrm{M}$

\begin{tabular}{llcccc}
\hline \hline Modelo & Ecuación del Modelo & P & $\begin{array}{c}\text { Sens } \\
\%\end{array}$ & $\begin{array}{c}\text { Esp } \\
\%\end{array}$ & $\begin{array}{c}\text { Precisión } \\
\%\end{array}$ \\
\hline I & Logit $=-7,5234+0,0752 * \operatorname{PrAc7}$ & 0,5 & 71,4 & 87,5 & 80,0 \\
II & Logit $=-31,4516+10,0218 *$ DPr7 & 0,5 & 85,7 & 100 & 93,3 \\
II & Logit $=-18,4701+0,0541 *$ It7 & 0,46 & 100 & 87,5 & 93,3 \\
\hline
\end{tabular}

Logit=ln(PrS/1-PrS), siendo PrS la probabilidad de ocurrencia de una epidemia severa (S) y ln es el logaritmo natural. Resolviendo la expresión $1 /\left(1+\right.$ Exp $\left.^{\text {-Logit }}\right)$ se obtiene el valor de PrS. Evento binario: PrM=1-PrS, siendo PrM probabilidad de observar una epidemia moderada a ligera. PrAc7: precipitación acumulada $(\mathrm{mm})$ del total de los registros de lluvia diaria $>7 \mathrm{~mm}$. DPr7: número de días con registro de precipitación $>7$ mm. It7=DPr7*PrAc7; 
de precisión de predicción). El modelo II registró un falso $M$, pues la severidad de las EFC de $42 \%$ (S) en Armstrong (campaña 2005/06), fue erróneamente predicha como moderada a ligera (M). Con el modelo III, solo la observación de severidad de la campaña 2005/06 de Cruz Alta clasificada con nivel epidémico moderado-ligero (M) fue mal predicha como severa (falso S). Las variables meteorológicas ligadas a la precipitación que integran los modelos descritos, fueron procesadas en el lapso comprendido entre las fechas de los estados fenológicos R3 y R5 (Tabla 1). Cuando se compararon los valores que alcanzan las variables PrAc7, DPr7 y IT7 en los percentiles $25 \%, 50 \%$ y $75 \%$, para el grupo de observaciones (7) con epidemias categorizadas como severas ( $\operatorname{SevEFC}>36 \%$ ), versus los valores de las mismas para el grupo (8) con niveles epidémicos moderados a ligeros ( $\operatorname{SevEFC}<=36 \%$ ), se observaron diferencias significativas entre las mismas (Figura 1).

En la mitad de los casos analizados con niveles epidémicos severos (S) se registraron (entre R3 y R5) cinco o más días con precipitaciones mayores a $7 \mathrm{~mm}$ y acumulados superiores a $127 \mathrm{~mm}$, casi duplicando lo observado en los casos con epidemias moderadas a ligeras (M). Las diferencias se incrementan cuando se analiza la componente interactiva It7 (508 $\mathrm{mm}$ versus $171,5 \mathrm{~mm}$ ).

\section{DISCUSIÓN}

Dentro del complejo de las EFC registradas en diversas campañas y variedades, $S$. glycines fue el patógeno predominante en comparación a los niveles encontrados de C. kikuchii. De esta forma la mancha marrón estuvo presente en la totalidad de los lotes evaluados, mientras que el tizón morado aproximadamente en la mitad de los mismos (prevalencia $53 \%$ ). Ambos patógenos son los más frecuentemente encontrados en la región pampeana causando síntomas foliares (Carmona et al., 2004). Tanto $S$. glycines como C. kikuchii son patógenos necrotróficos que son favorecidos por el monocultivo y la SD, condiciones de producción que se presentan en la mayoría de la superficie sembrada con soja en Argentina. A diferencia de lo que sucede en Brasil, Paraguay y Bolivia, donde la roya asiática causada por Phakopsora pachyrhizi S. \& P. Syd. genera preocupación permanente y es la principal enfermedad que orienta el control químico, en Argentina las EFC son las enfermedades de la soja mas prevalentes e intensas que impulsan y definen el control químico en este cultivo. Sin embargo, las aplicaciones de fungicidas no están basadas en criterios técnicos. De manera general la aplicación de fungicidas para EFC no sigue un criterio práctico basado en la estimación de la severidad, por el contrario, el criterio que prevalece es fito-céntrico basado en la fenología de la planta ya que se recomienda aplicar para EFC entre R3 a R5 sin considerar el nivel de enfermedad alcanzado (Hoffmann et al., 2004). Esta recomendación basada en el hospedante más que sobre el patógeno (Carmona et al.,

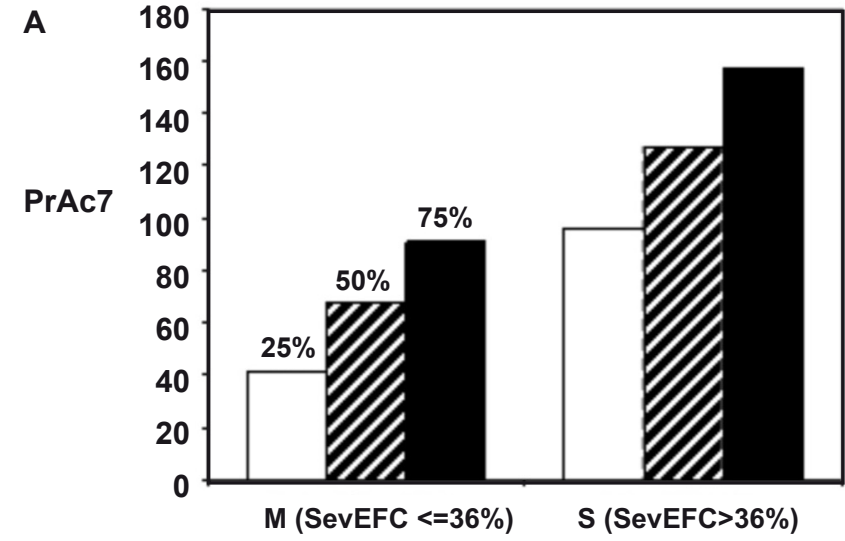

B

DPr7

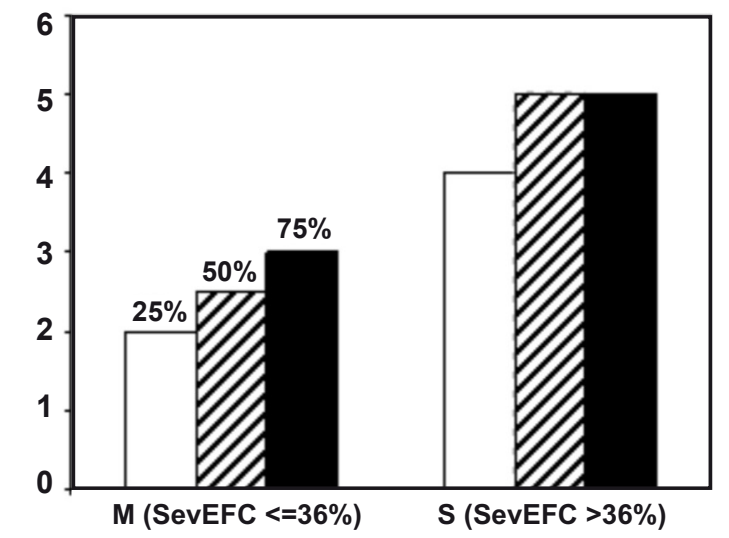

C

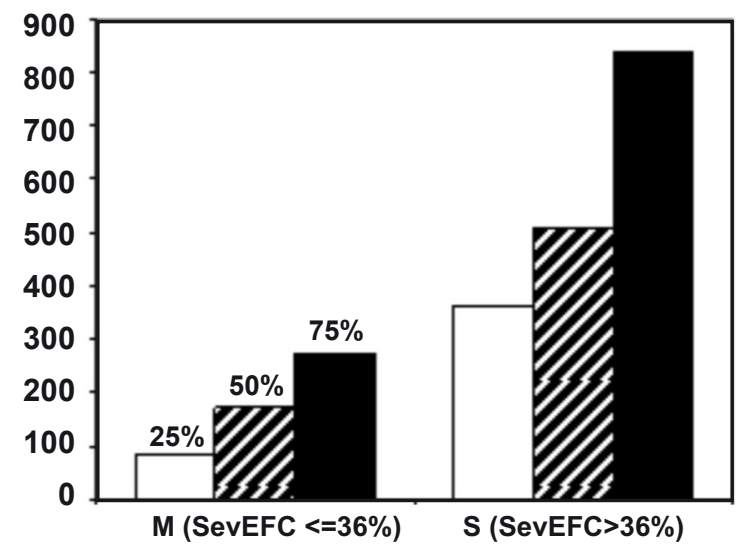

FIGURA 1 - Valores que alcanzan las variables meteorológicas individuales (A. PrAc7 y B. DPr7) e interactiva (C. It7), para el grupo de observaciones con niveles epidémicos severos $(\mathrm{S})$ y para el grupo con niveles moderados a ligeros $(\mathrm{M})$, en los percentiles 25,50 y $75 \%$.

2009), esta basada en el hecho de que estas enfermedades expresan sus síntomas mayormente al final de ciclo (de allí su nombre común) por lo que no existe una buena correlación entre la severidad en los estadios previos donde se deberían aplicar fungicidas (R3, R4, R5) y el impacto en los rendimientos (Martins, 2003; da Costa, 2005). Por todo esto, la estimación de la severidad final, observada en estadios como R6 y R7, representan un buen indicador para detectar diferencias o analizar epidemias y es por eso que 
esta medición (y no la severidad de estadios anteriores) es normalmente recomendada.

En este trabajo, las evaluaciones realizadas en el estadio R7 permitieron establecer diferencias entre los niveles epidémicos observados en diferentes años. La categorización aplicada del tipo de epidemias fue basada en la mediana, procurando distinguir fácilmente entre dos tipos de epidemias. Sin embargo, estudios futuros podrán establecer y mejorar con criterios biológicos y agronómicos, el tipo de clasificación basada por ejemplo en la relación entre severidad en algún estadio y los daños producidos.

Los niveles de severidad de las EFC registrados en R7, estuvieron significativamente asociados a las precipitaciones, principalmente a aquellas diarias que superaron el umbral de $7 \mathrm{~mm}$ durante R3 a R5. En concordancia, (Carmona et al., 2009) encontraron que entre 81 y $84 \%$ de la variación en la respuesta de rendimiento a la aplicación de fungicidas aplicados en R3 o en R5 respectivamente, fue explicada por la precipitación acumulada entre R3 y R5, siendo muy baja y no significativa la asociación con las lluvias entre R1 y R3. Los autores concluyeron que dicha respuesta estaría asociada a la mayor diseminación e infección para los principales patógenos observados en ese estudio (S. glycines, C. kikuchii, Colletotrichum truncatum, y Phomopsis sojae). En este mismo trabajo se analiza el impacto en el rendimiento por el uso de fungicidas en función de las lluvias, de esta manera con una cantidad de aproximadamente $120 \mathrm{~mm}$ ocurridos desde R3, se esperaría un impacto de alrededor de $400 \mathrm{~kg} \mathrm{ha}^{-1}$. Mientras que unos pocos milímetros menos $(100 \mathrm{~mm})$, el aumento en los rendimientos sería de $300 \mathrm{~kg} \mathrm{ha}^{-1}$. Los aportes de la presente investigación podrían enriquecer y mejorar la eficiencia de aquel modelo ya que por un lado confirma la importancia de las lluvias entre R3-R5 y por otro, establece que las lluvias de $7 \mathrm{~mm}$ o más, son las que deberían considerarse.

La lluvias actúan de dos maneras importantes para la diseminación de la enfermedad: 1) las gotas de lluvias remueven físicamente el inóculo a través del canopeo llevándolas desde tejidos enfermos o rastrojos infestados hacia plantas o tejidos sanos y 2) las lluvias proveen la humedad y horas de mojado necesarios para la germinación y penetración de los conidios. En este trabajo, tanto DPr7 como PrAc7 resultaron ser las variables meteorológicas simples más fuertemente correlacionadas con los niveles epidémicos. El sentido biológico de estas variables, se explica en el hecho de que lluvias que superan el umbral de $7 \mathrm{~mm}$ (excluyen lloviznas y lluvias ligeras) podrían asegurar una energía mínima para producir dispersión horizontal y vertical de conidios, muy requerido por los picnidios de $S$. glycines, predominante en el complejo bajo estudio. Con este mismo razonamiento es de suponer que los valores acumulados de esta cantidad de $\mathrm{mm}$ y su frecuencia, favorezcan a C. kikuchi para que se produzca la liberación, dispersión a corta distancia y se asegure el mojado necesario para que se inicie la infección (Tekrony et al., 1985; Schuh, 1993). Estas características permiten entender mejor la asociación entre las lluvias y la intensidad de las enfermedades observadas. Asimismo, muchos investigadores informaron respuestas eficientes e exitosas del uso de fungicidas dentro del período R3-R5, las cuales estuvieron fuertemente asociadas a las precipitaciones ocurridas desde R3 en adelante, lo que indicaría una relación con la mayor predisposición ambiental para los patógenos, (Backman et al., 1979, 1984; Pataky \& Lim, 1981a; Phillips, 1984; Carmona et al., 2008).

Respecto al género Septoria, sirve el ejemplo de $S$. tritici en trigo, donde la liberación de las picnidiosporas (o picnidioconidios) de los picnidios (infecciones secundarias) y su dispersión horizontal y vertical depende de la humedad y de la energía cinética de las gotas de lluvia. Hansen et al. (1994) encontraron una buena correlación entre la severidad de $S$. tritici y la frecuencia de días con más de $1 \mathrm{~mm}$ de lluvia durante 1 mes, comenzando en el estado fenológico de encañazón. En Argentina, Moschini (2007) halló una fuerte correlación entre la frecuencia de días con precipitaciones mayores a $7 \mathrm{~mm}$ y el coeficiente de infección de $S$. tritici, confirmando que a partir de dicho umbral $(7 \mathrm{~mm})$, es probable contar con la energía gravitacional y el mojado adecuados para la remoción y dispersión de las esporas de Septoria. Por el contrario, Lovell et al. (2004) demostró que la infección en las 3 hojas superiores de trigos de invierno podría ocurrir en ausencia de lluvias salpicantes, siendo mejor simulada por rocío. La arquitectura del canopeo afecta la proximidad de las hojas responsables del rendimiento en grano (niveles foliares superiores) al inóculo presente en hojas enfermas viejas dentro del cultivo. En esta situación, durante períodos de lluvias ligeras o rocío, la transferencia de conidios podría ocurrir a través del contacto directo de hojas emergentes con hojas enfermas (se solapan con las primeras). En el actual estudio no se contaron con registros de humedad relativa del aire, con los cuales se podrían haber procesado variables ligadas a la ocurrencia de mojado por rocío. No obstante, la proximidad de la siembra de las hileras de soja que permitirían el contacto entre hojas y entre plantas, no parece incrementar el nivel de intensidad de la mancha marrón causada por S. glycines (Pataky \& Lim, 1981b). Si bien existen algunas investigaciones para predecir el ataque de la mancha marrón basados en el ambiente, esta información se ha generado a través del estudio de combinaciones entre las horas de mojado y temperatura, pero no involucraron el abordaje desde la dispersión ni desde la cantidad de precipitaciones o días acumulados de lluvias (Schuh \& Adamowicz, 1993). Las variables DPr1 y PrAc1, que incluyen lloviznas y lluvias ligeras, no realizaron aportes extras significativos para ser incluidos en los modelos que estiman la probabilidad de los niveles epidémicos de las EFC aquí estudiadas. Probablemente lluvias de escasos milímetros no alcanzarían a remover el inóculo de $S$. glycines o a satisfacer las horas de mojado de C. kikuchii.

En este trabajo las variables hídricas analizadas fueron procesadas en lapsos comprendidos entre las fechas 
correspondientes a los estados fenológicos R3 y R5. La extensión de estos lapsos osciló entre 18 y 32 días (Tabla 1), variando en función de las características del cultivar y las condiciones térmicas de cada sitio. Otros enfoques posibles respecto al período de procesamiento de las variables ligadas a la lluvia pueden focalizarse en el uso de longitudes fijas en días o en grados día (temperatura base de la soja) a partir de R3. Preliminarmente, con los mismos datos utilizados en este estudio, cuando las variables hídricas se procesaron en lapsos fijos de 15, 20 y 25 días a partir de R3, se confirmó que las máximas correlaciones de Kendall se obtuvieron cuando se consideraron lluvias superiores a $7 \mathrm{~mm}$. Las correlaciones más altas correspondieron a períodos de 20 días a partir de R3, con valores de $0,73,0,60$ y 0,70 para las variables DPr7, PrAc7 y It7 respectivamente. Los modelos logísticos de respuesta binaria con las variables DPr7 y It7 alcanzaron precisiones de predicción de 93,3\%, similar a las obtenidas por los modelos de Tabla 3, que involucran a los mismos factores hídricos pero ajustados a los lapsos variables entre R3 y R5. Trabajos futuros podrán incursionar más profundamente en las alternativas planteadas respecto al período de análisis de las variables meteorológicas regresoras.

Los resultados del presente trabajo pueden servir como una base importante de un sistema de predicción para las principales enfermedades foliares que conforman las EFC. De esta manera, cuantificando de R3 a R5 las variables hídricas que mejor se ajustaron en este trabajo, sería posible predecir la intensidad de las manchas causadas por $S$. glycines y $C$. kikuchii en R7, categorizando un escenario epidémico severo o moderado a ligero. Asimismo, servirá para valorar que tipo y cantidad de precipitaciones (7 mm o más) son las que deberían considerarse para seguir los criterios de aplicación química para las EFC, recientemente recomendados (Carmona \& Reis, 2009). Más investigaciones que involucren aspectos agronómicos, genotípicos y otras variables ambientales, serán necesarias para desarrollar un sistema integral de predicción que pueda ser útil para el manejo químico de estas enfermedades.

\section{AGRADECIMIEINTOS}

El presente trabajo fue subsidiado por la Universidad de Buenos Aires (UBACyT G043).

\section{REFERENCIAS BIBLIOGRAFICAS}

Backman PA, Rodríguez Cabaña R, Hammond JM, Turlow DL (1979) Cultivar environment and fungicide effects on foliar disease losses in soybean. Phytopathology 69:562-564.

Backman PA, Crawford MA, Hammond JM (1984) Comparison of Meteorological and Standarized Timings of Fungicide Applications for Soybean Disease Control. Plant Disease 68:44-46.
Carmona M (2006) Importancia de las enfermedades de fin de ciclo: su relación con la ecofisiología y el uso estratégico de fungicidas en el cultivo de soja. Proceedings, Workshop de enfermedades de hoja, tallo y raíz. Mercosoja 2006, 3er Congreso de Soja del Mercosur. Rosário, Argentina. pp. 321-324.

Carmona MA, Gally M, Grijalba P, Sugia V, Jaeggi E (2004) Frequency and chemical control of causal pathogens of soybean late season diseases in the Pampeana Region. In: Abstracts of Contributed Papers and Posters. VII World Soybean Research Conference, IV International Soybean Processing and Utilization Conference. III Congresso Mundial de Soja. Foz de Iguazú PR, Brasil. p. 159.

Carmona M, Reis EM (2009) Sistema de Pontuação Para Aplicação de Fungicidas para as Doenças de Fim de Ciclo na Cultura da Soja. Proceedings, Seminário sobre critérios indicadores do momento para aplicação de fungicidas em Trigo e Soja. Passo Fundo RS. pp. 51-66.

Carmona M, Sautua F, Perelman S, Reis EM, Gally M (2009) Utilidad de las precipitaciones para predecir la respuesta al uso de fungicidas en el control de las EFC en soja. Anais, Mercosoja 2009, V Congreso de Soja del Mercosur, Goiana GO, CD Room ISBN 978857033-0130.

Díaz CG, Ploper LD, Galvez MR, Gonzalez V, Zamorano MA, Jaldo HE, Lopez C, Ramallo JC (2005) Effect of late season diseases on the growth of different soybean genotypes in relation to planting date. Agriscientia 22:1-7.

da Costa DIF (2005) Controle de doenças de final de ciclo na cultura da soja. Tese de Doutorado. Universidade Federal de Santa Maria. Santa Maria RS.

De Wolf ED, Madden LV, Lipps PE (2002) Risk assessment models for wheat fusarium head blight epidemics based in withinseason weather data. Phytopathology 93:428-435.

Fehr WR, Caviness CE, Burmood DT, Pennington JS (1971) Stage of development descriptions for soybeans Glycine max (L) Merrill. Crop Science 11:929-931.

Hansen JG, Secher BJM, Jorgensen LN, Welling B (1994) Thresholds for control of Septoria spp. in winter wheat based on precipitation and growth stage. Plant Pathology 43:183-189.

Hartman GL, Sinclair JB, Rupe JC (1999) Compendium of soybean diseases. 4th Ed. Saint Paul MN. APS Press.

Hoffmann LL, Reis EM, Forcelini CA, Panisson E, Mendes CS, Casa RT (2004) Efeitos da rotação de cultura, de cultivares e da aplicação de fungicida sobre o rendimento de grãos e doenças foliares em soja. Fitopatologia Brasileira 29:245-251.

Hughes G, Madden LV (2003) Evaluating predictive models with application in regulatory policy for invasive weeds. Agricultural Systems 76: 755-774.

Klingelfuss LH, Yorinori JT (2001) Infecção latente de Colletotrichum truncatum e Cercospora kikuchii em soja. Fitopatologia Brasileira 26:158-164.

Lim SM (1980) Brown spot severity and yield reduction in soybean. Phytopathology 70:974-977.

Lovell DJ, Parker SR, Hunter T, Welham SJ, Nichols AR (2004) Position of inoculum in the canopy affects the risk of septoria blotch epidemics in winter wheat. Plant Pathology 53:11-21.

Martins MC (2003) Produtividade da soja sob influência de 
ocorrência natural de Septoria glycines Hemmi e Cercospora kikuchii (Matsuo \& Tomoyasu) Gardner, com e sem controle químico. Tese de Doutorado. ESALQ, Universidade de São Paulo. Piracicaba SP.

Maude RD (1996) Seedborne diseases and their control. Principles and practice. Crop Protection 15:594.

Moschini RC, Sisterna MN, Carmona M (2006) Modelling of wheat black point incidence based on meteorological variables in the southern Argentinean Pampas Region. Australian Journal of Agricultural Research 57:1151-1156.

Moschini RC (2007) Los cultivos de Soja y Trigo: Herramientas para la Predicción y Manejo de enfermedades en la Argentina. Simposio "Mundo Agro 2007. Intensificación de los sistemas de producción y el manejo del riesgo en agricultura. Incorporando nuevas tecnologías al manejo de los cultivos". Buenos Aires. pp. $39-48$.

Niero AR, Tratch R, Pinto Junior AR (2007) Eficácia dos fungicidas no controle de doenças de final de ciclo (DFC) da soja. Revista Academica Curitiba 5:19-25.
Pataky JK, Lim SM (1981a) Efficacy of benomyl for controlling Septoria brown spot of soybean. Phytopathology 71:438-442.

Pataky JK, Lim SM (1981b) Effects of row width and plant growth habit on Septoria brown spot development and soybean yield. Phytopathology 71:1051-1056

Phillips DV (1984) Performance of foliar fungicide on soybeans in Georgia. Plant Disease 68:558-560.

Tekrony D, Stuckey R, Egli D, Tomes L (1985) Effectiveness of a point system for scheduling foliar fungicides in soybean seed fields. Plant Disease 69:962-965.

SAGPyA (2008) Estimaciones agrícolas mensuales. www.sagpya. mecon.gov.ar. SAGPyA - Secretaria de Agricultura, Ganadería, Pesca y Alimentación. Acesso em julio de 2009.

Schuh W (1993) Influence of interrupted dew periods, relative humidity, and light on disease severity and latent infections caused by Cercospora kikuchii on soybean. Phytopathology 83:109-113.

Schuh W, Adamowicz A (1993) Influence of assessment time and modeling on the relationship between temperature-leaf wetness periods and diseases parameters of Septoria glycines on soybeans. Phytopathology 83:941-948.

TPP 9098 - Recebido 10 Agosto 2009 - Acceptado 22 Abril 2010 Editor de Sección: Francisco F. Laranjeira 